\title{
Azimuthal Angular correlations in high energy processes in QCD
}

\author{
Jamal Jalilian-Marian ${ }^{1,2,3, a}$ \\ ${ }^{1}$ Baruch College, 17 Lexington Ave., New York, NY 10010, USA \\ ${ }^{2}$ CUNY Graduate Center, 365 Fifth Avenue, New York, NY 10016, USA \\ ${ }^{3}$ Centre de Physique Théorique, École Polytechnique, CNRS, Université Paris-Saclay, 91128 Palaiseau, \\ France
}

\begin{abstract}
Angular (azimuthal or polar) correlations between produced particles in high energy hadronic/nuclear collisions carry a wealth of information on the dynamics of the strong interactions in the novel regime where parton densities of the target hadron or nucleus are high. As such they probe a relatively unexplored region of QCD kinematic phase space. Here we consider azimuthal angular correlations between three jets produced in Deep Inelastic Scattering in the kinematics region corresponding to low $x_{\text {Bjorken }}$ of the target and show that high gluon density effects in the target lead to a disappearance of the away-side peaks.
\end{abstract}

\section{Introduction}

Deep Inelastic Scattering (DIS) of a lepton (electron, positron, $\cdots$ ) on a hadron (proton, $\cdots$ ) or nucleus is the cleanest environment in which to probe the dynamics of the strong interactions between the fundamental constituents of matter, quarks and gluons. The scattering cross section can be factorized into two parts; the electromagnetic part where the incoming lepton radiates a virtual photon (for large enough virtualities one must include exchange of other electroweak bosons such as $Z$ ) which then couples to the quarks in the target proton/nucleus. The electromagnetic part of the interaction is governed by QED and is well understood. The interaction of the virtual photon with the quarks in the hadron can be written in terms of two structure functions (if one does not care about polarization effects) $F_{1}$ and $F_{2}$ or equivalently $F_{2}$ and $F_{L}$.

These structure functions contain all the information about dynamics of strong interactions and are therefore highly non-trivial (and non-perturbative). The depend on two independent variables $x_{B j o r k e n} \equiv \frac{-q^{2}}{2 P \cdot q}$ where $q$ is the 4-momentum of the exchanged photon with virtuality $Q^{2}=-q^{2}$ and $P$ is the target hadron 4-momentum. It can be shown that $x_{B j o r k e n}=x$ where $x$ is the fraction of energy of the hadron carried by an individual quark. The photon virtuality $Q^{2}$ can also be thought of the renormalization scale of the bilinear quark operator corresponding to number of the quarks in the hadron (quark distribution function). The corresponding gluon distribution function, counting the number of gluons in the hadron wave function (in light cone gauge) is extracted from the quark distribution function via scaling violation. It is an experimental fact that the number of (sea) quarks and gluons increases very fast as one probes smaller values of $x$. In fact the gluon distribution function is

\footnotetext{
ae-mail: jamal.jalilian-marian@baruch.cuny.edu
} 
predicted to grow power-like at small $x$. This fast growth leads to the unpleasant fact that perturbative cross sections obtained from these parton distribution functions would eventually violate perturbative unitarity. Therefore one expects new dynamics of QCD to emerge at small $x$ which would tame this power growth.

\section{QCD at small $x$ : gluon saturation}

Even before the experimental observation of the fast growth of parton distribution functions, and specially gluon distribution function, the question of what tames this fast growth had been considered by Gribov, Levin and Ryskin (GLR) [1] who proposed the idea of gluon saturation. At small enough $x$ the number density of gluons becomes so large that the probability for a gluon to interact with neighboring gluons becomes as large as the standard gluon radiation in perturbative QCD. The new mechanism is typically referred to as recombination and becomes significant when the probability

$$
\frac{\alpha_{s}}{Q^{2}} \frac{x G\left(x, Q^{2}\right)}{\pi R^{2}} \sim 1
$$

. Here the first factor is the effective partonic scattering cross section and the second factor is the number of gluons per unit area, i.e., density or packing factor. They proposed a new and non-linear evolution equation which takes this re-interaction into account. Mueller and Qiu (MQ) [2] then derived this non-linear equation using Feynman diagram techniques in PQCD. The GLR-MQ equation reduces to the standard (linear) DGLAP evolution equation in the high $Q^{2}$ (low density) limit and has the nice feature that it leads to a slow down of the gluon distribution function, commonly referred to as gluon saturation.

The phenomenon of gluon saturation in QCD was put on a more formal footing using the an effective action approach first proposed by McLerran and Venugopalan (MV) [3]. The essence of the MV approach is the emergence of a new semi-hard (or even hard) scale called saturation scale $Q_{s}$. Very roughly, the saturation scale is the momentum scale at which the relation 1 is satisfied. This scale depends (and grows) with $x$ and also depends on the nucleon number $A$ and the impact parameter of the collision $b_{t}$. Based on theoretical studies and comparisons with experimental data its value is given by

$$
Q_{s}^{2}\left(x, b_{t}, A\right)=1 G e V^{2} A^{1 / 3}\left(\frac{x_{0}}{x}\right)^{\lambda}
$$

with $x_{0}=3 \times 10^{-4}, \lambda \sim 0.28-0.3$ and $A^{1 / 3} \sim 5-6$ for most central collisions off a large nucleus such as gold or lead ([4] for reviews). The MV effective action is based on separation of large $x$ and small $x$, or equivalently on fast and slow, degrees of freedom. This is possible due to the vastly different natural time scales natural to small $x$ and large $x$ partons. The large $x$ partons of a fast proton or nucleus look frozen (due to time dilation) to the small $x$ partons which are thought of as fast fluctuations. Due to the large number of small $x$ gluons it is more efficient to represent them as one coherent classical field rather than an ensemble of non-interacting partons. In the MV model the classical field $A_{c l}^{\mu}$ represents the small $x$ gluons and can be obtained by solving the QCD classical equations of motion with a color current $J^{\mu}$ representing the large $x$ degrees of freedom such as valence quarks and large $x$ sea-quarks and gluons. In the MV model the color current has only one large component due to proton or nucleus moving almost at the speed of light. The classical equations of motion are then easy to solve (in terms of the color charge $\rho$ ) and can be shown to have the following form (in light cone gauge $A^{-}=0$ )

$$
\begin{aligned}
& A_{a}^{+} \sim \frac{1}{\partial_{\perp}^{2}} \rho_{a} \\
& A_{\perp}=0
\end{aligned}
$$


where $a$ is the color index. One calculates observables in this background field and finally averages of all color charges $\rho_{a}$ with some distribution probability. In the original MV model the distribution of color charges is taken to be a Gaussian, a good model for a large nucleus, of the form

$$
F\left[\rho_{a}\right] \equiv \exp \left[-\frac{\rho_{a}^{2}\left(x_{t}\right)}{\mu^{2}}\right] .
$$

This "weight function" $F\left[\rho_{a}\right]$ then serves as the initial condition for JIMWLK evolution equation which determines how the distribution of color charges changes with energy (or $x$ ). The MV effective action approach can be understood as being equivalent to pQCD in a background field of the type given in eq. (3).

The effective degrees of freedom in applications of the MV effective action to particle production in high energy hadronic/nuclear collisions [5] are quark and gluon propagators in this background field. For example, to calculate the QCD structure functions $F_{2, L}$ in this approach, one needs the quark propagator given by

$$
S_{F}(p, q) \equiv S_{F}^{0}(2 \pi)^{4} \delta^{4}(p-q)+S_{F}^{0} \tau_{F}(p, q) S_{F}^{0}(q)
$$

where

$$
S_{F}^{0}(p)=\frac{i(p+m)}{p^{2}-m^{2}+i \epsilon}
$$

is the free fermion propagator and $\tau_{F}(p, q)$ represents the interactions (multiple scatterings) of the quark with the color field of the the target hadron or nucleus. The gluon propagator in this background field is needed when one considers gluon production and is given by

$$
G_{\mu \nu}^{a b}(p, q) \equiv G_{\mu \nu}^{0 a b}(p) \delta^{a b}(2 \pi)^{4} \delta^{4}(p-q)+G_{\mu \lambda}^{0}(p) \tau_{g}^{a b}(p, q) G_{\nu}^{0, \lambda}(q)
$$

where $G_{\mu \nu}^{0, a b}$ is the free gluon propagator,

$$
G_{\mu \nu}^{0, a b}(k)=\frac{i d_{\mu \nu}(k)}{k^{2}+i \epsilon} \delta^{a b}
$$

with the projector

$$
d_{\mu v}(k) \equiv\left[-g_{\mu v}+\frac{k_{\mu} n_{v}+k_{v} n_{\mu}}{n \cdot k}\right]
$$

and $\tau_{F}, \tau_{g}$ contain the all-order scattering from the target color field, defined via

$$
\begin{aligned}
& \tau_{F}(p, q) \equiv(2 \pi) \delta\left(p^{-}-q^{-}\right) \gamma^{-} \int d^{2} x_{t} e^{i\left(p_{t}-q_{t}\right) \cdot x_{t}}\left\{\theta\left(p^{-}\right)\left[V\left(x_{t}\right)-1\right]-\theta\left(-p^{-}\right)\left[V^{\dagger}\left(x_{t}\right)-1\right]\right\} \\
& \tau_{g}(p, q) \equiv-(2 \pi) \delta\left(p^{-}-q^{-}\right) 2 p^{-} \int d^{2} x_{t} e^{i\left(p_{t}-q_{t}\right) \cdot x_{t}}\left\{\theta\left(p^{-}\right)\left[U\left(x_{t}\right)-1\right]-\theta\left(-p^{-}\right)\left[U^{\dagger}\left(x_{t}\right)-1\right]\right\}
\end{aligned}
$$

where $V, U$ are Wilson lines in the fundamental and adjoint representation of $S U\left(N_{c}\right)$ and re-sums all the re-scatterings of the quark and gluon from the background color field,

$$
V\left(x_{t}\right) \equiv \hat{P} \exp \left\{\operatorname{ig} \int d x^{-} A_{a}^{+}\left(x^{-}, x_{t}\right) t_{a}\right\}
$$

and

$$
U\left(x_{t}\right) \equiv \hat{P} \exp \left\{i g \int d x^{-} A_{a}^{+}\left(x^{-}, x_{t}\right) T_{a}\right\}
$$


and $t_{a}$ and $T_{a}$ are the corresponding generators. The approximation made in deriving these expressions is the high energy approximation, also commonly referred to as the eikonal approximation (see also [6] for quark propagator in a more general setting).

The QCD structure functions at small $x$ can then be written in a factorized form as a convolution of two independent parts; the probability for a virtual photon to split into a quark anti-quark pair (called a dipole) before it reaches the target and the probability for this dipole to scatter from the color field of the target. The first part is determined from QED while the second part contains all the QCD dynamics. This can be written as

$$
F_{\perp, L}\left(x, Q^{2}\right)=\sigma_{0} \int d^{2} r_{t} d z\left|\Psi_{\perp, L}\right|^{2}\left(z, Q^{2}\right) N\left(x, r_{t}\right)
$$

where $z$ is the fraction of the virtual photon energy carried by the quark and $r_{t}$ is the transverse separation between the quark and anti-quark. Here we have integrated over the transverse profile of the target which gives $\sigma_{0}$. The dipole-target scattering total cross section $N$ (imaginary part of the forward scattering amplitude) is given in terms of Wilson lines (in fundamental representation) $V\left(x_{t}\right)$ as

$$
N\left(x, r_{t}\right)=\frac{1}{N_{c}} \operatorname{Tr}\left(1-V\left(x_{t}\right) V^{\dagger}\left(y_{t}\right)\right)
$$

and $r_{t} \equiv x_{t}-y_{t}$ is the dipole size with $b_{t}=\frac{x_{t}+y_{t}}{2}$ integrated to give $\sigma_{0}$. At the classical level the dipole cross section $N$ does not depend on $x$ (is energy independent), rather the $x$ dependence come via the JIMWLK [7] evolution equation. In the large $N_{c}$ limit this evolution equation is known as the BK [8] equation and can be written as

$$
\frac{d}{d \log 1 / x}=\frac{N_{c} \alpha_{s}}{2 \pi^{2}} \int d^{2} z_{t} \frac{\left(x_{t}-y_{t}\right)^{2}}{\left(x_{t}-z_{t}\right)^{2}\left(y_{t}-z_{t}\right)^{2}}\left[N\left(x_{t}, z_{t}\right)+N\left(z_{t}, y_{t}\right)-N\left(x_{t}, y_{t}\right)-N\left(x_{t}, z_{t}\right) N\left(z_{t}, y_{t}\right)\right]
$$

The BK equation has very interesting properties; in the dilute limit where $N \ll 1$ one can ignore the non-linear term and it reduces to the BFKL equation. In this limit the scattering probability grows with energy very fast (power-like) and one approaches the dense region. On the other hand, the nonlinear term is essential in the dense region where $N \sim 1$, and the solution of BK equation describing the scattering probability approaches one, i.e., perturbative unitarity is restored. The solution also exhibits geometric scaling, i.e., observables depend on combination of dipole size $r_{t}$ and the saturation scale $Q_{s}$ rather than depending on each variable separately. The approximate forms of the solution to BK equation in different kinematic regions can be written in momentum space as follows,

$$
\begin{array}{ll}
N\left(p_{t}\right) \sim \frac{1}{p_{t}^{2}}\left[\frac{Q_{s}^{2}}{p_{t}^{2}}\right]^{\gamma} & p_{t} \gg Q_{s} \\
N\left(p_{t}\right) \sim \frac{1}{p_{t}^{2}}\left[\frac{Q_{s}^{2}}{p_{t}^{2}}\right]^{\gamma} & p_{t} \geq Q_{s} \\
N\left(p_{t}\right) \sim \log \left[\frac{Q_{s}^{2}}{p_{t}^{2}}\right] & p_{t} \leq Q_{s} .
\end{array}
$$

where $\gamma<1$ is related to BFK anomalous dimension. This approximate form shows the most essential property of the solution to BK equation, the change in growth of the scattering probability with energy (or $\mathrm{x}$ ) keeping in mind that the saturation scale depends on $x$. This form also makes it clear that when one constructs the so-called nuclear modification factor $R_{p A} \sim \frac{\sigma^{p A}}{\sigma^{p p}}$ this ratio can be different from 1 (since $Q_{s}^{2}$ is different for protons and nuclei) in some kinematics regions signifying presence of 
nuclear effects. The same dipole scattering probability appears in proton structure functions for which there is a wealth of data from HERA. It is now believed that this effective action approach can describe the proton structure function data at $x \leq 10^{-2}$ and for a wide range of photon virtuality $Q^{2}$.

This dipole (and its adjoint counterpart describing scattering of gluons) scattering probability appears in many physical observables in particle production in high energy collisions involving hadron and nuclei, for example in single inclusive particle production in high energy proton-nucleus collisions at RHIC and the LHC. One thus compare predictions of this effective theory of small $x$ QCD with experimental results and put severe constraints on the various inputs that go into initial conditions of the theory.

For less inclusive quantities such di-jet production in DIS or pA collisions more complicated structures appear in the cross section, namely the four-point function of Wilson lines, referred to as the quadrupole operator $Q$. This operator also obeys [9] the JIMWLK equation and in the large $N_{c}$ approximation can be written in terms of dipoles, albeit in a complicated manner. Nevertheless it can be shown that all these higher point operators asymptotically grow with energy [9] the same way as the dipole so that one expects all physical observables to grow with energy in a similar way. It can also be shown that the JIMWLK evolution equation for these higher point functions reduces to the BJKP equation in the linear limit [10].

In this contribution we will focus on production of three partons, a quark, an anti-quark and a gluon that is radiated from either the quark or anti-quark [11] in DIS,

$$
\gamma^{\star} p(A) \rightarrow q \bar{q} g
$$

It is possible for the produced partons to scatter from the target either before or after the gluon radiation. Scattering of a given parton from the target is described as propagation of the parton in a color background field where the quark and gluon propagators are given in eqs. $(5,7)$.

The amplitude for production of a quark, an anti-quark and a gluon then can be written as

$$
\begin{aligned}
i \mathcal{A}_{1}= & (i e)(i g) \int \frac{d^{4} k_{1}}{(2 \pi)^{4}} \bar{u}(p) \gamma^{\mu} t^{a} S_{F}\left(p+k, k_{1}\right) \gamma^{\nu} S_{F}\left(k_{1}-l,-q\right)\left[S_{F}^{(0)}(-q)\right]^{-1} v(q) \epsilon_{v}(l) \epsilon_{\mu}^{*}(k), \\
i \mathcal{A}_{2}= & (i e)(i g) \int \frac{d^{4} k_{1}}{(2 \pi)^{4}} \bar{u}(p)\left[S_{F}^{(0)}(p)\right]^{-1} S_{F}\left(p, k_{1}\right) \gamma^{v} S_{F}\left(k_{1}-l,-q-k\right) \gamma^{\mu} t^{a} v(q), \epsilon_{v}(l) \epsilon_{\mu}^{*}(k) \\
i \mathcal{A}_{3}= & (i e)(i g) \int \frac{d^{4} k_{1}}{(2 \pi)^{4}} \int \frac{d^{4} k_{2}}{(2 \pi)^{4}} \bar{u}(p)\left[S_{F}^{(0)}(p)\right]^{-1} S_{F}\left(p, k_{1}-k_{2}\right) \gamma^{\lambda} t^{c} S_{F}^{(0)}\left(k_{1}\right) \gamma^{v} \\
& S_{F}\left(k_{1}-l,-q\right)\left[S_{F}^{(0)}(-q)\right]^{-1} v(q)\left[G_{\lambda}^{\delta}\right]^{c a}\left(k_{2}, k\right)\left[G_{\delta}^{(0), \mu}(k)\right]^{-1} \epsilon_{v}(l) \epsilon_{\mu}^{*}(k) \\
i \mathcal{A}_{4}= & (i e)(i g) \int \frac{d^{4} k_{1}}{(2 \pi)^{4}} \int \frac{d^{4} k_{2}}{(2 \pi)^{4}} \bar{u}(p)\left[S_{F}^{(0)}(p)\right]^{-1} S_{F}\left(p, l-k_{1}\right) \gamma^{v} S_{F}^{(0)}\left(-k_{1}\right) \gamma^{\lambda} t^{c} \\
& S_{F}\left(k_{2}-k_{1},-q\right)\left[S_{F}^{(0)}(-q)\right]^{-1}\left[G_{\lambda}^{\delta}\right]^{c a}\left(k_{2}, k\right)\left[G_{\delta}^{(0) \mu}(k)\right]^{-1} \epsilon_{v}(l) \epsilon_{\mu}^{*}(k)
\end{aligned}
$$

where $\epsilon_{\nu}(l), \epsilon_{\mu}^{*}(k)$ respectively denote polarization vectors of the virtual photon and the outgoing gluon. Transverse momentum of the virtual photon is set equal to zero so that $l^{+}=-Q^{2} / 2 l^{-}$without any loss of generality. Also, $p^{+}$is the light cone energy of the incoming quark and $q^{+}$is the light cone energy of the final state quark. To proceed further it is convenient to use spinor helicity formalism to evaluate the numerators involving Dirac matrices. To do so one needs to choose and work wit a given helicity of the particle involved. For example, contribution of the first term above which describes scattering 
of the quark and anti-quark with the target before the quark radiates a gluon can be written as

$$
\begin{aligned}
i \mathcal{A}_{1}^{a}= & \operatorname{ieg} \int d^{4} x d^{4} y \frac{d^{4} k_{1}}{(2 \pi)^{4}} e^{-i\left(p+k-k_{1}\right) x} e^{-i\left(k_{1}-l+q\right) y} \delta\left(x^{+}\right) \delta\left(y^{+}\right) \\
& t^{a}\left[\theta\left(p^{+}+k^{+}\right) V\left(x_{t}\right)-\theta\left(-p^{+}-k^{+}\right) V^{\dagger}\left(x_{t}\right)\right]\left[\theta\left(-q^{+}\right) V\left(y_{t}\right)-\theta\left(q^{+}\right) V^{\dagger}\left(y_{t}\right)\right] \\
& \frac{N_{1}}{(p+k)^{2} k_{1}^{2}\left(k_{1}-l\right)^{2}}
\end{aligned}
$$

and $N_{1}$ is the numerator containing the Dirac matrices,

$$
N_{1} \equiv \bar{U}(p) \notin^{\star}(k)(\not p+\not k) h k_{1} \notin(l)\left(\not k_{1}-\not\right) h V(q)
$$

so that the Dirac part is contained in $N_{1}$. To illustrate spinor helicity methods we consider the case when the virtual photon is longitudinal whereas the quark has positive helicity (this fixes the antiquark to have negative helicity due to helicity conservation) and gluon also has positive helicity. This is denoted $N_{1}^{L ;+-+}$ and is given by

$$
N_{1}^{L ;+-+}=-\frac{\sqrt{2}}{[n k]} \frac{Q}{l^{+}}[n p]<k p>[n p]<n \bar{k}_{1}>\left[n \bar{k}_{1}\right]<n q>\left(<n \bar{k}_{1}>\left[n \bar{k}_{1}\right]-l^{+}<n \bar{n}>[n \bar{n}]\right) .
$$

Each factor above is a simple kinematics factor, for example $<n q>\sim q^{+}$and so on except for $<k p>$ which is slightly more involved (however it is canceled by an identical factor in the denominator of the amplitude).

Similarly, the contribution of the third term in the amplitude which describes radiation of a gluon from the quark and the subsequent scatterings of the quark, anti-quark and gluon system from the target can be written as

$$
\begin{aligned}
i \mathcal{F}_{3}^{a}= & e g \prod_{j=1}^{3} \int d^{2} \mathbf{x}_{j} \int \frac{d^{4} k_{1} d^{4} k_{2}}{(2 \pi)^{5}} e^{i\left(\mathbf{k}_{1}-\mathbf{k}_{2}-\mathbf{p}\right) \cdot \mathbf{x}_{1}} e^{-i\left(\mathbf{k}_{1}+\mathbf{q}\right) \cdot \mathbf{x}_{2}} e^{i\left(\mathbf{k}_{2}-\mathbf{k}\right) \cdot \mathbf{x}_{3}} \delta\left(p^{+}-k_{1}^{+}+k_{2}^{+}\right) \\
& \delta\left(k_{1}^{+}-l^{+}+q^{+}\right) \delta\left(k_{2}^{+}-k^{+}\right) V\left(x_{t}\right) t^{b} V^{\dagger}\left(y_{t}\right) U\left(z_{t}\right)^{b a} \\
& \frac{\left(-2 k^{+}\right) N_{3}}{\left[\left(k_{1}-k_{2}\right)^{2}+i \epsilon\right]\left[k_{1}^{2}+i \epsilon\right]\left[k_{2}^{2}+i \epsilon\right]\left[(k-l)^{2}+i \epsilon\right]},
\end{aligned}
$$

with $N_{3}$ defined as

$$
N_{3}^{\lambda_{\gamma} ; \lambda_{q} \lambda_{\bar{q}} \lambda_{g}}=\bar{u}_{\lambda_{q}} \not h\left(k_{1}-\not k_{2}\right)\left(\notin^{\left(\lambda_{g}\right)}\right)^{*}\left(k_{2}\right) \not k_{1} \notin^{\left(\lambda_{\gamma}\right)}(l)\left(\not k_{1}-l\right) h v_{\lambda_{\bar{q}}}(q) .
$$

Here $\lambda_{\gamma, q, \bar{q}, g}$ refer to polarizations of the virtual photon, quark, anti-quark and gluon respectively. It is possible to further simplify the Dirac numerators by factorizing them into smaller pieces which can be evaluated once and used everywhere. For example, one can write

$$
\begin{aligned}
& N_{1}^{\lambda_{\gamma} ; \lambda_{q} \lambda_{\bar{q}} \lambda_{g}}=Q_{\gamma^{*} \rightarrow q \bar{q}}^{\lambda_{\gamma} \lambda_{q}}\left(\bar{k}_{1}\right) \cdot Q_{q \rightarrow q g}^{\lambda_{q} \lambda_{g} ; 1}\left(\bar{k}_{1}\right) \\
& N_{3}^{\lambda_{\gamma} ; \lambda_{q} \lambda_{\bar{q}} \lambda_{g}}=Q_{\gamma^{*} \rightarrow q \bar{q}}^{\lambda_{\gamma} \lambda_{q}}\left(\bar{k}_{1}\right) \cdot Q_{q \rightarrow q g}^{\lambda_{q} \lambda_{g} ; 3}\left(\bar{k}_{1}\right)+\frac{k_{1}^{2}}{2 k_{1}^{+}} \cdot Q_{\gamma^{*} \rightarrow q \bar{q}}^{\lambda_{\gamma} \lambda_{q}}(n) \cdot Q_{q \rightarrow q g}^{\lambda_{q} \lambda_{g} ; 3}(n),
\end{aligned}
$$

where

$$
\begin{aligned}
Q_{\gamma^{*} \rightarrow q \bar{q}}^{\lambda_{\gamma} \lambda_{q}}\left(\bar{k}_{1}\right) & =\bar{u}_{\lambda_{q}}\left(\bar{k}_{1}\right) \notin^{\left(\lambda_{\gamma}\right)}(l)\left(k_{1}-l\right) h v_{\lambda_{\bar{q}}}(q) \\
Q_{q \rightarrow q g}^{\lambda_{q} \lambda_{g} ; 1}\left(\bar{k}_{1}\right) & =\frac{1}{(p+k)^{2}} \cdot \bar{u}_{\lambda_{q}}(p)\left(\notin^{\left(\lambda_{g}\right)}\right)^{*}(k)(p+\not k) h u_{\lambda_{q}}\left(\bar{k}_{1}\right) \\
Q_{q \rightarrow q g}^{\lambda_{q} \lambda_{g} ; 3}\left(\bar{k}_{1}\right) & =\bar{u}_{\lambda_{q}} \not h\left(k_{1}-\not k_{2}\right)\left(\notin^{\left(\lambda_{g}\right)}\right)^{*}\left(k_{2}\right) u_{\lambda_{q}}\left(\bar{k}_{1}\right) .
\end{aligned}
$$


For example, for the splitting $\gamma^{*} \rightarrow q \bar{q}$ with configuration where the incoming photon has longitudinal polarization $(\lambda=L)$ we get

$$
\begin{aligned}
Q_{\gamma^{*} \rightarrow q \bar{q}}^{L+}\left(\bar{k}_{1}\right) & =\frac{Q}{l^{+}}\left[\bar{k}_{1} n\right]\left(\left\langle n \bar{k}_{1}\right\rangle\left[\bar{k}_{1} n\right]-\langle n \bar{l}\rangle[\bar{l} n]\right)\langle n q\rangle, \\
& =-4 \frac{Q}{l^{+}}\left(p^{+}+k^{+}\right)^{\frac{1}{2}}\left(q^{+}\right)^{\frac{3}{2}} \\
Q_{\gamma^{*} \rightarrow q \bar{q}}^{L+}(n) & =0 \\
Q_{\gamma^{*} \rightarrow q \bar{q}}^{L-}\left(\bar{k}_{1}\right) & =\left(Q_{\gamma^{*} \rightarrow q \bar{q}}^{L+}\left(\bar{k}_{1}\right)\right)^{*}=Q_{\gamma^{*} \rightarrow q \bar{q}}^{L+}\left(\bar{k}_{1}\right)
\end{aligned}
$$

The results for other terms and polarizations are similar. One also has products of Wilson lines which can be organized as products of dipoles, dipole times a quadrupole and quadrupole squared as the leading $N_{c}$ terms. More complicated structure appear as sub-leading $N_{c}$ contributions. To evaluate there products one can either solve the JOMWLK equation for them to obtain a solution or use the leading $N_{c}$ approximation to write all these terms of products of dipoles. The rest of the calculation proceed in a similar fashion and can be used to make predictions for azimuthal angular distribution of three partons (jets) produced in high energy DIS. The most interesting result is that the away side peaks disappear due to presence of gluon saturation. We refer the reader to [11] for full details.

\section{References}

[1] L. V. Gribov, E. M. Levin and M. G. Ryskin, Phys. Rept. 100, 1 (1983). doi:10.1016/03701573(83)90022-4

[2] A. H. Mueller and J. w. Qiu, Nucl. Phys. B 268, 427 (1986). doi:10.1016/0550-3213(86)90164-1

[3] L. D. McLerran and R. Venugopalan, Phys. Rev. D 49, 2233 (1994) doi:10.1103/PhysRevD.49.2233 [hep-ph/9309289]; S. Jeon and R. Venugopalan, Phys. Rev. D 70, 105012 (2004) doi:10.1103/PhysRevD.70.105012 [hep-ph/0406169]; A. Dumitru, J. JalilianMarian and E. Petreska, Phys. Rev. D 84, 014018 (2011) doi:10.1103/PhysRevD.84.014018 [arXiv:1105.4155 [hep-ph]].

[4] H. Weigert, Prog. Part. Nucl. Phys. 55, 461 (2005); F. Gelis, E. Iancu, J. Jalilian-Marian, R. Venugopalan, Ann. Rev. Nucl. Part. Sci. 60, 463-489 (2010). [arXiv:1002.0333 [hep-ph]]; J. JalilianMarian, Y. V. Kovchegov, Prog. Part. Nucl. Phys. 56, 104-231 (2006); J. L. Albacete and C. Marquet, Prog. Part. Nucl. Phys. 76, 1 (2014).

[5] A. Dumitru and J. Jalilian-Marian, Phys. Rev. Lett. 89, 022301 (2002); D. Kharzeev, Y. V. Kovchegov and K. Tuchin, Phys. Rev. D 68, 094013 (2003); J. L. Albacete, N. Armesto, A. Kovner, C. A. Salgado and U. A. Wiedemann, Phys. Rev. Lett. 92, 082001 (2004); J. Jalilian-Marian, Y. Nara and R. Venugopalan, Phys. Lett. B 577, 54 (2003) doi:10.1016/j.physletb.2003.09.097; J. Jalilian-Marian, J. Phys. G 30, S751 (2004), Nucl. Phys. A 748, 664 (2005), J. Jalilian-Marian, Nucl. Phys. A 753, 307 (2005) doi:10.1016/j.nuclphysa.2005.02.156, Phys. Rev. C 70, 027902 (2004), Nucl. Phys. A 739, 319 (2004); A. Dumitru, A. Hayashigaki and J. Jalilian-Marian, Nucl. Phys. A 765, 464 (2006), Nucl. Phys. A 770, 57 (2006); E. M. Henley and J. Jalilian-Marian, Phys. Rev. D 73, 094004 (2006) doi:10.1103/PhysRevD.73.094004; F. Gelis and J. Jalilian-Marian, Phys. Rev. D 76, 074015 (2007), Phys. Rev. D 67, 074019 (2003) doi:10.1103/PhysRevD.67.074019, Phys. Rev. D 66, 094014 (2002) doi:10.1103/PhysRevD.66.094014, Phys. Rev. D 66, 014021 (2002) doi:10.1103/PhysRevD.66.014021; J. Jalilian-Marian and A. H. Rezaeian, Phys. Rev. D 85, 014017 (2012), J. Jalilian-Marian and A. H. Rezaeian, Phys. Rev. D 86, 034016 (2012); A. H. Rezaeian, 
Phys. Rev. D 86, 094016 (2012); J. Jalilian-Marian and Y. V. Kovchegov, Phys. Rev. D 70, 114017 (2004) [Erratum-ibid. D 71, 079901 (2005)]; A. Kovner and M. Lublinsky, JHEP 0611, 083 (2006), Phys. Rev. D 84, 094011 (2011), Phys. Rev. D 83, 034017 (2011); J. Jalilian-Marian, Nucl. Phys. A 770, 210 (2006); C. Marquet, Nucl. Phys. A 796, 41 (2007); J. L. Albacete and C. Marquet, Phys. Rev. Lett. 105, 162301 (2010).

[6] J. Jalilian-Marian, Phys. Rev. D 96, no. 7, 074020 (2017) doi:10.1103/PhysRevD.96.074020 [arXiv:1708.07533 [hep-ph]].

[7] J. Jalilian-Marian, A. Kovner, L. D. McLerran and H. Weigert, Phys. Rev. D 55, 5414 (1997); J. Jalilian-Marian, A. Kovner, A. Leonidov and H. Weigert, Nucl. Phys. B 504, 415 (1997), Phys. Rev. D 59, 014014 (1999), Phys. Rev. D 59, 014015 (1999), Phys. Rev. D 59, 034007 (1999), A. Kovner, J. G. Milhano and H. Weigert, Phys. Rev. D 62, 114005 (2000); A. Kovner and J. G. Milhano, Phys. Rev. D 61, 014012 (2000); E. Iancu, A. Leonidov and L. D. McLerran, Nucl. Phys. A 692, 583 (2001), Phys. Lett. B 510, 133 (2001); E. Ferreiro, E. Iancu, A. Leonidov and L. McLerran, Nucl. Phys. A 703, 489 (2002).

[8] I. Balitsky, Nucl. Phys. B 463, 99 (1996); Y. V. Kovchegov, Phys. Rev. D 60, 034008 (1999); Phys. Rev. D 61, 074018 (2000).

[9] A. Dumitru and J. Jalilian-Marian, Phys. Rev. D 82, 074023 (2010), Phys. Rev. D 81, 094015 (2010); A. Dumitru, J. Jalilian-Marian, T. Lappi, B. Schenke and R. Venugopalan, Phys. Lett. B 706, 219 (2011); A. Dumitru, K. Dusling, F. Gelis, J. Jalilian-Marian, T. Lappi and R. Venugopalan, Phys. Lett. B 697, 21 (2011) doi:10.1016/j.physletb.2011.01.024;

[10] A. Ayala, E. R. Cazaroto, L. A. Hernández, J. Jalilian-Marian and M. E. Tejeda-Yeomans, Phys. Rev. D 90, no. 7, 074037 (2014) doi:10.1103/PhysRevD.90.074037; J. Jalilian-Marian, Phys. Rev. D 85, 014037 (2012) doi:10.1103/PhysRevD.85.014037.

[11] A. Ayala, M. Hentschinski, J. Jalilian-Marian and M. E. Tejeda-Yeomans, Phys. Lett. B 761, 229 (2016) doi:10.1016/j.physletb.2016.08.035, Nucl. Phys. B 920, 232 (2017) doi:10.1016/j.nuclphysb.2017.03.028 [arXiv:1701.07143 [hep-ph]]. 